KÁROLY RÉDEI

\title{
Bemerkungen zu zwei mißlungenen etymologischen Versuchen
}

The author of the article refutes two etymologies proposed by $\mathrm{H}$. $\mathrm{Katz}$, on phonetic grounds. The nationality name jugra (PP jógra) cannot be linked etymologically with Zyryan jegir 'swamp forest, swamp' etc. since an original $\ddot{a}$ must be assumed for the vowel of the first syllable (cf. UEW 93: *jä $\eta k \ddot{a}$ 'Moor, Sumpf...' FU). Nor is it possible to link the Finnish word perkele 'devil' with Germanic *dwergila-z etc. because the Germanic sequence $d w e$ - can correspond to Finnish ve- but not pe-.

1. Syrjänisch jegra, Russisch jugra

1. Wie bekannt stammt der von fremden Völkern gebrauchte Name der Ungarn (lat. (h)ungarus, russ. vengr usw.) aus slaw. ${ }^{*} g g(b) r$-, welche Form wiederum auf die bulgarisch-türkische Stammesverbandbenennung onogur (on 'zehn' + ogur 'Stammesname') zurückgeht. Mit diesem Namen hat man das Wort Jugra, jugor in Zusammenhang gebracht, welches Wort sich in den alten russischen Quellen auf die Obugrier bezieht bzw. ihr Wohngebiet bezeichnet. Die ältesten altrussischen Belege (jugra, jugor, jugry) kommen in der Kiewer Urchronik (Nestor-Chronik) aus dem 11. 
Jahrhundert vor, darauffolgend wird das Wort jugra usw. in den russischen Annalen sehr häufig erwähnt. Es erhebt sich nun die Frage, ob das auf die Ungarn bezügliche slaw. ${ }^{\circ g}($ b) $r$ - < bulg.türk. onogur mit dem Namen der Obugrier jugra gleichzustellen ist. M. Zsirai (Finnugor népnevek. I. Jugria. Budapest 1930 passim) hat die zwei Formen miteinander verglichen und jugra auf eine frühere russische Form ugra zurückgeführt. Das $j$ in jugra erklärt er mit russischer Präjotierung. Die etymologische Gleichstellung der beiden Formen ist jedoch mit mehreren Schwierigkeiten verbunden: 1. Die die Ungarn bezeichnende Form lautet folgerichtig mit einem Vokal an, das Wort jugra beginnt in der überwiegenden Mehrheit der Fälle mit $j$ (auch die arabisch-persischen Quellen kennen die Form mit $j$-: Jüra, $J u g r a)$. 2. Ein weiteres Problem liegt darin, daß die Obugrier niemals zum Stammesverband der Onoguren gehörten. 3. Zu Beginn des onogurischen Stammesverbandes in der südrussischen Steppe um das 5. Jahrhundert lebten die Wogulen und Ostjaken teilweise schon in Westsibirien, teilweise noch an der europäischen Seite des Urals (vgl. Hajdú: in Hajdú \& Domokos, Die uralischen Völker und Sprachen. Hamburg 1987, 29, 47-49). Angesichts dieser Schwierigkeiten haben einige Forscher versucht, neuere etymologische Erklärungen zu bieten (s. Hajdú: a. a. O.). Von den verschiedenen Theorien ist die von I. Vásáry am wahrscheinlichsten. Er bringt den Namen Jugria mit uigurischen Volksbewegungen in westliche Richtung in Zusammenhang. Seiner Meinung nach stammt der Name jugra aus dem Namen eines uigurischen Stammes Yugur. Diese Hypothese harrt aber noch weiterer Beweise (in: A. Róna-Tas [ed.], Chuvash Studies. Budapest 1982, 247-257).

2. Kürzlich haben sich $\mathrm{H}$. Katz und Ch. Koch (UAJb. NF 7 (1987): 99-104) mit der Benennung (J)Ugra auseinandergesetzt. Der Berliner Slawist Ch. Koch hat die altrussischen Angaben zum Wort jugra, ugra überprüft und ist zu dem Ergebnis 
gekommen, daß die Schreibung mit $u$ - sekundär ist, und diejenige mit ju- (jugra, jugry) somit die ältere Form vertritt (99-102). Katz baut seine neue Etymologie auf diese Feststellung. Er nimmt an, daß russ. jugra eine syrjänische Entlehnung ist: (Wied.) jegra (jogra, jugra) 'Ostjake, Wogule', (WUo.) V jegra '»vielleicht Wogule» (sagt Cember)', (Fokos-Fuchs) V (selten) jegra 'Ostjake', (SrSIK) VO Vm. VU Peč. jegra 'ханты и манси'1. Katz leitet syrj. jegra aus dem Substantiv Ud. SO jegir, VL jegir 'Sumpfwald, Sumpf' her. Die ursprüngliche Bedeutung der mit dem Adjektivsuffix $a$ versehenen Form wäre demnach 'Sumpfwäldler' gewesen. Diese semantisch übrigens tadellos scheinende Erklärung scheitert an lautlichen Aspekten. Für den Völkernamen jegra nimmt auch Katz das Rekonstrukt PP *jogra an. Er weiß weiterhin, daß syrj. jegir, jegir 'Sumpfwald, Sumpf' in UEW (93) unter *jänkä 'Moor, Sumpf... ' (FU) eine sichere Etymologie hat. Einer solchen FU Grundform könnte nur PP * jögir entsprechen. Katz (103): „doch ist das Rekonstrukt [*jänkä] fürs Lapp. zumindest ungünstig, da $a$ in aller Regel auf ${ }^{*} e$ zurückzuführen ist (Korhonen, Johdatus 84, 88 f.) “. Diese Äußerung von Katz beinhaltet jedoch einen groben Irrtum! Vorlapp. ${ }^{*} e$ hat eine Fortsetzung $a$, wenn in der zweiten Silbe frühurlapp. * $\dot{a}$ oder ${ }^{*} \rho(<* \ddot{a})$ gestanden hat; der Typ fi. elä- 'leben' lapp. alle- gehört hierher (Korhonen 84). Bei Korhonen (88) steht, daß dem vorlapp. $\ddot{a}$ lapp. ie entspricht, wenn in der zweiten Silbe ein frühurlapp. ${ }^{*} e\left(<{ }^{*}\right)$ gestanden hat; der Typ von fi. käte- (käsi) 'Hand' lapp. giettâ gehört hierher. Lapp. jag'ge 'bog, marsh' paßt zu der unter Punkt 3) gesagten Regel (Korhonen 89): vorlapp. ${ }^{*} \ddot{a}>$ urlapp. ${ }^{*} \varepsilon>a$ usw. vor dem Vokal $*^{*} a$ (od. * $*^{\circ}<\ddot{a}$ ) in der zweiten Silbe (Typ fi. päivä 'Tag, Sonne' lapp. bri've). Katz hätte also den Passus Korhonens weiterlesen müssen, um eine Antwort auf seinen Zweifel zu finden.

Zugunsten seiner wankenden Etymologie nimmt Verf. eine sekundäre Palatalisierung $*_{e}>e$ nicht nur im lappischen, sondern 
auch im wogulischen und ostjakischen Glied der Wortsippe

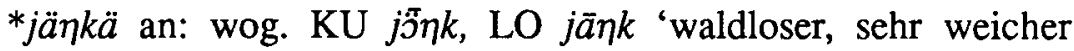
Sumpf' < urwog. *jä $\eta k \sim$ ostj. \ jē $\eta k$ usw. 'offene, baumlose Stelle auf dem Moor' < obugr. *jē $\eta k$ (vgl. Honti, GOV 143). Die sekundäre Palatalisierung eines velaren Vokals unter dem Einfluß des anlautenden $j$ ist freilich möglich. Es ist aber schwer zu glauben, daß dieser Prozeß im Falle der einwandfreien etymologischen Sippe *jä $\eta \ddot{a}$ in allen Sprachen bzw. Sprachgruppen (Lapp., Perm., Obugr.!) vor sich gegangen wäre. Und all diese Mühen im Interesse, den schwer etymologisierbaren Völkernamen syrj. jegra, russ. jugra aus dem Sumpf herauszuziehen.

3. Der Einfall, eine Volksbenennung aus einem Wort 'Sumpf' zu deuten, hat dem Verf. so sehr gefallen, daß er noch weitere Benennungen (suomalainen, Finne, Lappi) auf diese Weise zu erklären versucht hat. Von diesen werde ich mich nur mit dem Namen suomi, suomalainen auseinandersetzen, da diese Etymologie für die mit mehreren Fehlern beladene Arbeitsmethode von Katz besonders kennzeichnend ist. Er meint, daß die von Stiernhielm (aus dem Jahre 1600) stammende Erklärung von Suomi, suomalainen aus dem Kompositum suo-maa 'Sumpf-Land' das Richtige getroffen hat. Er will suo-maa aus einer hypothetischen Form *sōye-mā > *sōyema, ,mit Anpassung von * $m \bar{a}$ an den damals möglichen Nebensilbenvokalismus" herleiten (103). Das Rekonstrukt *sōye enthält zwei Fehler: 1. Fi. suo geht sicherlich auf eine zweisilbige frühurfinnische Form zurück, jedoch dürfte der inlautende Konsonant nicht nur $\gamma$, sondern genauso auch $j, \eta$ und $w$ gewesen sein; 2 . der lange Vokal ist durch den Schwund des inlautenden Konsonanten entstanden. Also: *soye / ?*soje / *sone / *sowe > *sō (> suo). Im Falle des angenommenen *sōye-

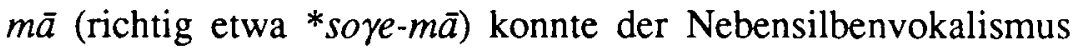
nicht zu einem kurzen Vokal führen, weil es sich hier um ein für das Sprachgefühl gut analysierbares Kompositum handelt. Die von Katz angegebene Entwicklungsreihe *sōyema > *sōyen (Gen. 
*sōyema-n) > *sōyen, *sōyemen > *sōn, *sōmen, daher Suomi, suomen (durch Anpassung an den Typ lumi, lumen) widerspricht unseren Kenntnissen über die finnische Lautgeschichte. Eine hypothetische Form *soyema hätte nur folgende Prozesse durchmachen können: *soyema $>*_{\text {soyemi }} \gg *_{\text {soin, }}(\mathrm{Gen}.){ }^{*}$ soimen. Vgl. seinämä 'Wand' > seinä(i)n, -men, seinin, -imen (vgl. Hakulinen, SKRK ${ }^{4}$ 130-132); ebenso bei dem deverbalen Nominalsuffix -mal-mä: *kantama > kannin, -ntimen, kannoin, -ntoimen 'Tragriemen', *pukema > puin, pukimet 'Kleidungsstücke', *luoma > loin : loinpuut ( luomapuut) 'Scher-, Zettelrahmen' (op. cit. 193-195, s. auch 42-43). Die von Katz erwähnte Alternative - freilich mit kurzem Vokal in der ersten Silbe *soyema $>$ *soyemi > *sōmi > suomi ( suoma-) wäre theoretisch möglich; diese Erklärung scheitert jedoch daran, daß die Abkürzung *soye-

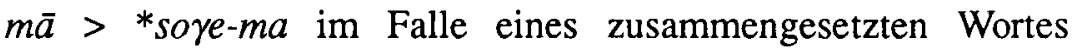
auszuschließen ist. Zu der richtigen Etymologie von Suomi, suomalainen $\mathrm{s}$. SKES.

Katz leitet die Völkernamen Finne und Lappi, lappalainen auch aus 'Sumpf, Pfuhl' bedeutenden Wörtern ab. In diesem Zusammenhang möchte ich auf die Lemmata Lappi, vatja, vepsä in SKES hinweisen, aus denen deutlich wird, daß diese Benennungen - wie auch noch vuowjos 'Lappe' und Finne - eine einheitliche semantische Gruppe bilden: alle diese Wörter haben ursprünglich 'Keil' oder 'Flosse' bedeutet. Der Namengebung liegt die Tatsache zugrunde, daß etliche ostseefinnische und lappische Stämme keilförmige (dreieckige) Stoffstïckchen als Kennzeichen oder Amulette auf ihren Kleidern trugen. Dieses Amulett haben die Stämme auch als Warenzeichen verwendet. Später begannen diese Wörter die Mitglieder des dasselbe Amulett tragenden Stammes bzw. Volkes zu bezeichnen. Zu diesem Typ gehört auch die Benennung Karjala, karjalainen (Pekkanen: Vir. 1984: 191-193). 
2. Finnisch perkele 'Teufel'

1. Nach einer in der finnisch-ugrischen Fachliteratur allgemein verbreiteten Ansicht, ist das finnische Wort perkele 'Teufel' wahrscheinlich ein baltisches Lehnwort (Kalima, Itämerensuomalaisten kielten balttilaiset lainasanat 1936, im weiteren IKBL 147; SKES). Diese Etymologie wird von H. Katz (UAJb. NF 8 (1988): 8-13) abgelehnt, seine Gegenargumente werden weiter unten auseinandergesetzt. Zunächst möchte ich jedoch seinen neuen etymologischen Vorschlag prüfen.

Katz leitet fi. perkele 'Teufel', estn. pörgu 'Hölle' aus dem Germanischen her: urgerm. *dwerga- (an. dvergr, dt. Zwerg). Er meint, dem osfi. *perkeleš (> perkele) stehe die germanische Deminutivform *dwergila-z am nächsten; estn. pörgu < *perko- $i$ (evtl. <*perka-i) stammt aus germ. *dwerga-, *dwergo-.

Diese Etymologie stößt auf gravierende Schwierigkeiten. Mit einiger Mühe könnte man die semantische Entwicklung noch erklären ('Zwerg' $\rightarrow$ 'Wicht, Kobold' $\rightarrow$ 'Teufel'), die lautlichen Probleme sind jedoch unüberbrückbar. Bei der Lautsubstitution germ. $* d w$ - bzw. ${ }^{*} w$ - $>$ osfi. ${ }^{*} p$ - nimmt er auf die in der etymologischen Literatur vorbildliche Monographie T. Hofstras (Ostseefinnisch und Germanisch. Groningen 1985) Bezug, interpretiert allerdings das dort Gelesene völlig falsch. Germ. ${ }^{*} w$ - wird durch osfi. $p$ - nur vor $\ddot{u}$ (= fi. $y$ ) ersetzt: pyrkiä 'streben', py(y)hkiä 'wischen', pyhä 'heilig' (Hofstra 74; s. auch Koivulehto: Vir. 1973: 13). Vor allen anderen Vokalen kommt als Substitution von germ. $*_{w}$ - immer osfi. $v$-vor (Hofstra $74-75$ ). Wegen des anlautenden Konsonantismus können fi. perkele, estn. pörgu den urgerm. $* d$ wergila-z, *dwerga- nicht entsprechen.

Für die Lautsubstitution germ. ${ }^{*} w$-, ${ }^{*} k w-,{ }^{*} \chi w-,{ }^{*} t w->$ osfi. ${ }^{*} p$ - gibt Katz (10-11) weitere sechs Etymologien an. Hofstra 
(a. a. O.) behandelt in seinem Buch auch die Konsonantenverbindungen, deren zweiter und letzter Konsonant ein $w$ ist. Hier kommen folgende Lautentsprechungen zum Vorschein: germ. ${ }^{*} s w$ - $>$ osfi. $h$-, $v$-, germ. ${ }^{*} k w$-, ${ }^{*} \chi w->$ osfi. $k$-, $v$-, germ. ${ }^{*} p w->$ osfi. $v$-, jedoch niemals $p-$ ! Man könnte freilich annehmen, daß Katz die Substitutionsmöglichkeiten der germanischen Lehnwörter im Osfi. weiterentwickeln wollte. Dies ist ihm jedoch nicht gelungen, da die vorgebrachten Etymologien außer der unmöglichen Lautentsprechung germ. ${ }^{*} w$-, Konsonant $+{ }^{*} w$ - $>$ osfi. $p$ auch anderweitigen Problemen begegnen.

Fi. (Lönnr.) pale 'Erdrücken (weit kleiner als ein Landrücken)' $\left(\leftarrow\right.$ nwgerm. $\left.{ }^{*} \chi w \bar{a} l a-z\right)$ geht möglicherweise auf eine frühere Form *pa $\delta$ zurück und kann somit etymologisch mit fi. pade 'Weg, Pfad' (s. SKES) zusammengehören.

Fi. pika- 'schnell' $\leftarrow$ urgerm. *kwikwa-: man sollte eine finnische Form *kika oder *vika erwarten.

Fi. pysyä, dial. pisyä '(ver)bleiben, sein' $\leftarrow$ urgerm. *wesan $>$ got. wisan '(da)sein'. Das finnische Wort ist von FW Herkunft und hat Entsprechungen im Tscher. und Mord. (SKES; UEW 732: *pise- 'bleiben, verbleiben...'). Fi. $y$ in der zweiten Silbe ist ein Reflexivsuffix. Zu dem Lautwandel $i>\ddot{u}(y)$ in der ersten Silbe s. UEW.

Fi. parka 'elend' $\leftarrow$ urgerm. *wraka $>$ mndl. wrac 'untauglich, verdorben', afries. wrac 'beschädigt'. Fi. parka 'arm, unglücklich, elend' hat in den Dialekten auch die Bedeutung 'Wickelzeug, Windel, Lappen' (SKES). In diesem Fall hat sich ein 'Kleidungsstück' bedeutendes Wort zum Ausdruck des Mitleides entwickelt (s. auch rukka, rääsy, SKES). Die obige Zusammenstellung ist also sowohl in lautlicher wie auch semantischer Hinsicht abzulehnen.

Fi. piha 'Hof' $\leftarrow$ urgerm. *wixsa- > got. veihs 'Flecken, Dorf'. Das finnische Wort ist FP Ursprungs: *piča 'Pfahl, Stange' FP (UEW). Zu der herkömmlichen Etymologie fügt Katz folgendes 
hinzu: „Die übliche Zusammenstellung des fi. Wortes mit tscher. KB pitša 'Einzäunung, Gehege' scheitert am konsonantischen Inlaut: *- ${ }_{-}->$fi. $-t-"(10-11)$. Diese Feststellung stimmt keinesfalls. FU ${ }^{*}-\check{c}$ - kann im Fi. wie bekannt die Fortsetzungen $-t$ - und $-h$ - haben (zu dieser Frage s. zuletzt Rédei: FUM 12/13 (1988(89): 63-67). Für die etymologische Arbeitsmethode von Katz ist folgendes Verfahren charakteristisch: nach fi. piha 'Hof' wird der Hinweis „ma. 'Dorf' (SKES 541)“ angegeben. In SKES steht aber, daß die Bedeutung 'kylä; Dorf' nur in einer lokalen Mundart (Kuortane) bekannt ist. Er mißt also dieser offenbar sekundären und marginalen Bedeutung bei der Etymologisierung eine entscheidende Wichtigkeit bei.

Estn. piht (gen. piha, pihe) 'Stange' $\leftarrow$ urgerm. *twista-(z), vgl. mengl. twist 'Zweig'. Das estnische Wort ist eine einwandfreie Entsprechung von fi. piha 'Hof' $\leftarrow$ 'aus Pfählen gemachter Zaun' $\leftarrow$ 'Pfahl, Stange' (vgl. SKES; UEW 729). In der Fußnote 15 liest man, „daß germ. *twista-(z) ein einwandfreies Modell für fi. vihta 'Zweigbündel', vihdas 'Birkenreisig' abgibt (vgl. zum Wort SKES 1739)“. Laut SKES ist es unsicher, ob das finnische Wort aus dem Russischen stammt. Diese eventuelle Herkunft wird von Katz weder abgetan noch überhaupt erwähnt.

Koivulehtos und Hofstras These steht also fest: germ. $*_{w-}>$ osfi. $v$-, (vor $\ddot{u}) p$-. Das urgerm. ${ }^{*}-w$ - im Inlaut hat normalerweise auch eine Substitution $v$ - im Osfi. (Hofstra 103). „Urgerm. /w/ wird in 12 Lehnwörtern nicht durch stufenwechselloses /v/, sondern durch urfi. /p/ (d. h. $p$ im Stufenwechsel mit $\beta>$ fi. $v$ ) reflektiert." (Hofstra: a. a. O.) Z. B.: arpi (arven) 'Narbe', häpeä 'Schande, Schmach' hävetä 'sich schämen' usw. In einigen germanischen Lehnwörtern kommt ein Wechsel $p \sim v$ vor: rapea / ravea 'rasch', hipiä / hiviä 'Teint, Gesichtsfarbe' (Hofstra 104). $\mathrm{Zu}$ der Unterstützung der Etymologie germ. *dwerga-, *dwergo- > estn. pörgu, *dwergila-(z) > osfi. *perkeleš $>$ fi. perkele sucht Katz auch bei der inlautenden Vertretung des germ. 
$*_{-w-} \rightarrow$ osfi. $p \sim v$ Zuflucht. Diese Zuflucht ist aber aussichtslos, weil sie die lautlich und semantisch auch sonst völlig unsichere Etymologie nicht schützen kann: wie Hofstra schön und überzeugend bewiesen hat, kann osfi. $p$ nur im Stufenwechsel mit $v$ den urgerm. Spiranten $*$ - $w$ - ersetzen.

Urgerm. * $w$ - kann im Osfi. ausnahmsweise als Reflex $v \sim m$ haben, z. B.: havaita hamata 'bemerken' (Koivulehto: Vir. 1974: 113, Anm. 2; Hofstra 104). Hier haben wir es wahrscheinlich mit einer osfi. sporadischen Lautentwicklung $v>m$ zu tun. Vgl. auch helme $\sim$ helve 'Spelze' < urgerm. * $x e l w \bar{o}(n)$-. Neben der Variante mit $m$ ist immer eine mit $v$ vorhanden.

Bei Hofstra gibt es also kein Beispiel für die Lautsubstitution urgerm. ${ }^{*}-w->$ osfi. $-m-$. Abgesehen von den klar dargestellten Bedingungen des Vorkommnisses $v \sim m$ an der Stelle des urgerm.

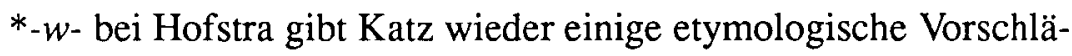
ge, in denen dem urgerm. ${ }^{*}$-w- im Osfi. $m$ (also ohne einen Wechsel mit $v$ !) entsprechen würde (11-12). Mit diesen, anscheinend ad hoc-Etymologien werde ich mich hier nicht auseinandersetzen. Nur eine kurze Bemerkung: fi. armas 'lieb, lieblich' wird nicht aus urgerm. *arwa-z, sondern aus *armaz hergeleitet.

Katz will auch fi. piru 'böser Geist, Teufel' (dial. peru) aus frühurgerm. ${ }^{*} d w e r g o$ - herleiten. Diese Erklärung scheitert wiederum an der bereits dargestellten unwahrscheinlichen Lautsubstitution urgerm. ${ }^{*} d w$ - $>$ osfi. $p$-. Nach SKES dürfte fi. piru (peru) eine von den zahlreichen euphemistischen Verdrehungen von perkele sein.

2. Wie stehen wir nun zu dem baltischen Ursprung von fi. perkele 'Teufel'? Ist er wirklich abzulehnen, wie es Katz tut? Sehen wir zuerst die wichtigsten Angaben zu dieser Wortsippe: fi. perkele 'Teufel, Satan', kar. perkeleh, pergeleh id.; wot. perkele id. (< fi.); estn. (dial.) pergel, perkläne, pärgel, pörgel, pärgal id.; pörgu 'Hölle'. Die wichtigsten euphemistischen Verdrehungen im Fi.: perkala, perkula, parkule, perskele, pirkele, pirskule usw. 
Kalima (IKBL 147) leitet diese osfi. Wortsippe aus dem Baltischen her: lit. perkūnas 'Donnergott des heidnischen Altertums; Donner', perkúnija 'Donner', lett. pērkūns, pērkuôns 'Donner', apreuss. percunis id. Die lautliche Seite der Etymologie ist tadellos. Die baltischen Formen lit. perkúnas, perkúnija, lett. pērkūns usw. haben die Ostseefinnen als abgeleitete Wörter aufgefaßt. Auf diese Weise hat man einerseits eine osfi. Form *perku (> estn. *pörgu) rückgebildet (s. auch Kalima: a. a. O.), andererseits hat man die baltischen Segmente -nas, -nija, -ns mit dem eigenen Ableitungssuffix -leš substituiert: *perkeleš > perkeleh > perkele. Die Beziehung der Formen *perku > estn. põrgu und *perkeles ( > perkele) läßt sich dadurch leicht erklären, daß es im Finnischen viele Ableitungen auf -ale / -äle / -ele gibt, die häufig über Varianten auf $-u /-y$ verfügen. Z. B.: järkäle järky, ketale kettu, riepale $\sim$ riepu (vgl. Hakulinen, SKRK ${ }^{4} 163$ ). Das erste $e$ des Suffixes ele (vgl. kyynele, sävele usw.) erklärt das zweite $e$ von perkele statt des aufgrund des Baltischen zu erwartenden $u$ (vgl. jedoch perkula, parkule). $\mathrm{Zu}$ den Bedeutungen balt. 'Donnergott, Donner' osfi. 'Teufel' vgl. aind. deváh 'himmlisch, göttlich, Gott' aw. daēvō 'Daēva, ,alter Gott“, Dämon', neupers. dēv 'Dämon' 'Gott' (Mayrhofer, KEWA 2: 63-64). Was schließlich das semantische Verhältnis 'Donnergott' 'Teufel' anbelangt, dürfte in Erinnerung gebracht werden, daß der Teufel und die bösen Geister nach der jüdischen, christlichen und mohammedanischen Religion gefallene Engel sind. Die estnische Bedeutung ('Hölle') kann man aufgrund der Bedeutungsänderung 'Teufel' $\rightarrow$ 'Unterwelt' $\rightarrow$ 'Hölle' gut verstehen.

3. Aus dem Gesagten geht hervor, daß fi. perkele kein Zwerglein war. Urgerm. *dwerga - hat im Finnischen allerdings einen Niederschlag gefunden und zwar in folgenden zwei Wörtern: *verka-: (Lönnr.) verkanen 'förbannad varelse, hin onde; verdammtes Wesen, Teufel, der Böse', verkasen poika 'förbannad karl; verdammter Kerl' < *dwerga- (an. dvergr, schwed. dvärg, 
dt. Zwerg); *turka: turka, turkka 'armseliger, krüppelhafter, ungestalter Mensch; etw. Häßliches, Abscheuliches', turkanen 'Teufel' ? < urgerm. *durga- > nd. dorf 'Zwerg'; *durga- wird als die tiefstufige Variante von *dwerga- angesehen (Tunkelo: Vir. 1911: 53-57; SKES; Katz: a. a. O. 13). Die germanische Herkunft von turka, turkanen ist aber unsicher, da es auch ein eigenwüchsiges Wort sein dürfte.

\section{Anmerkung}

1 Falls sich Vásárys Etymologie aus dem uigurischen Stammesnamen als richtig erweist, könnte syrj. jegra < PP *jg̈gra ein Lehnwort aus uig. yugur (in unserer Transkription: jugur) sein. In diesem Fall handelt es sich bei syrj. $a$ um das bekannte Adjektivsuffix (Bedeutung etwa 'dem Stamm jugur gehörig'). Die lautliche Entsprechung ist regelmäßig: vorperm. * $u$ hat in mehreren Beispielen eine PP Fortsetzung * $\dot{\phi}$, z. B.: wotj. S kej, K $k o j$ 'Speck, Schmalz, Talg' < *kuje 'Fett' FU (UEW 195); wotj. S mid, K mâd 'anderer' syrj. S P męd, PO mùd id. < *mu 'ander(er)' FU (UEW 281); wotj. S G tẹl, K tól 'Wind' syrj. S tẹl, PO tül id. < *tule 'Wind' FP (UEW 800); wotj. S G ess, $\mathrm{K}$ os 'Tür' syrj. es : eş̧ss id. < *ukse 'Tür' FP (UEW 803). Unter dieser Voraussetzung dürfte russ. jugra aus frühurperm. * jugra entlehnt worden sein. Syrj. (Wied.) jogra, jugra ist möglicherweise eine Rückentlehnung aus dem Russischen. 
